quatrième série-tome 46 fascicule 6 novembre-décembre 2013

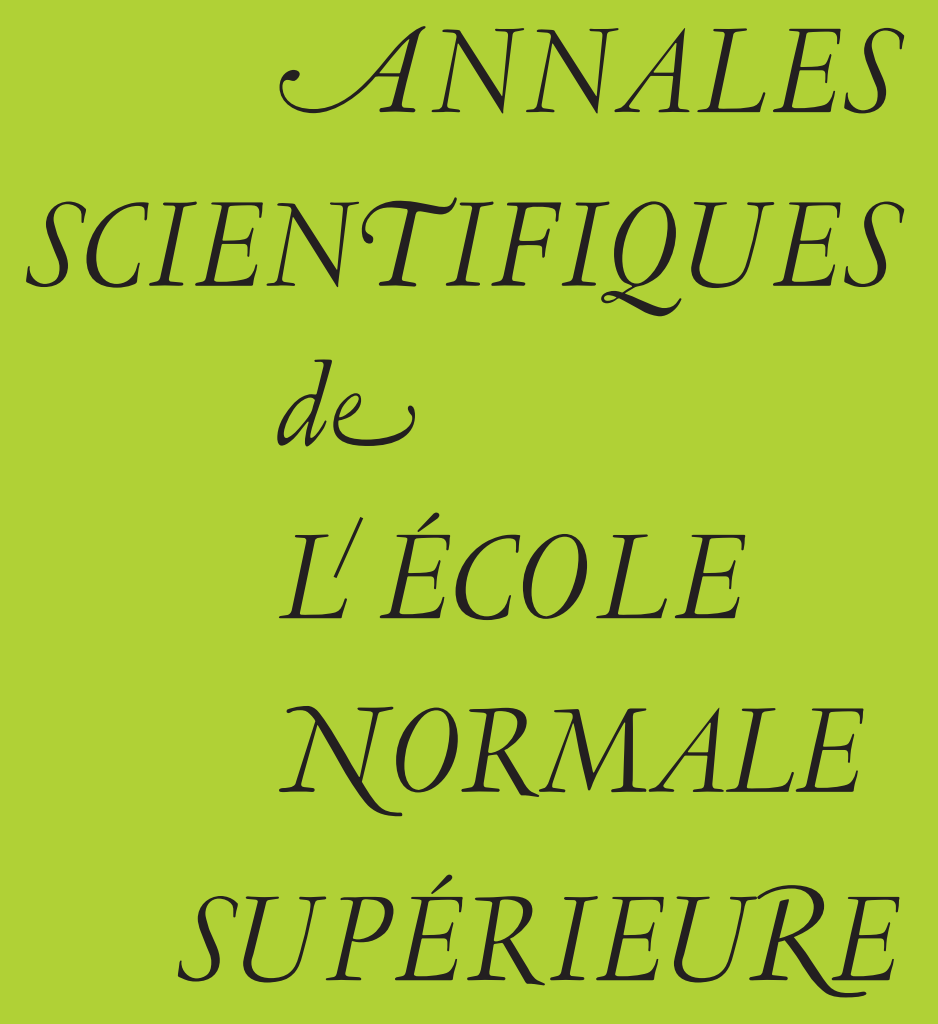

\title{
Yann BUGEAUD
}

Automatic continued fractions are transcendental or quadratic 
Ann. Scient. Éc. Norm. Sup.

$4^{\mathrm{e}}$ série, t. 46, 2013, p. 1005 à 1022

\title{
AUTOMATIC CONTINUED FRACTIONS ARE TRANSCENDENTAL OR QUADRATIC
}

\author{
BY YANN BUGEAUD
}

\begin{abstract}
We establish new combinatorial transcendence criteria for continued fraction expansions. Let $\alpha=\left[0 ; a_{1}, a_{2}, \ldots\right]$ be an algebraic number of degree at least three. One of our criteria implies that the sequence of partial quotients $\left(a_{\ell}\right)_{\ell \geq 1}$ of $\alpha$ is not 'too simple' (in a suitable sense) and cannot be generated by a finite automaton.

RÉsumé. - Nous établissons de nouveaux critères combinatoires de transcendance pour des développements en fraction continue. Soit $\alpha=\left[0 ; a_{1}, a_{2}, \ldots\right]$ un nombre algébrique de degré au moins égal à trois. L'un de nos critères entraîne que la suite $\left(a_{\ell}\right)_{\ell \geq 1}$ des quotients partiels de $\alpha$ n'est pas trop simple (en un certain sens) et ne peut pas être engendrée par un automate fini.
\end{abstract}

\section{Introduction and results}

A well-known open question in Diophantine approximation asks whether the continued fraction expansion of an irrational algebraic number $\alpha$ either is ultimately periodic (this is the case if, and only if, $\alpha$ is a quadratic irrational), or contains arbitrarily large partial quotients. As a preliminary step towards its resolution, several transcendence criteria for continued fraction expansions have been established recently [1, 4, 5, 9] (we refer the reader to these papers for references to earlier works, which include [23, 14, 27, 12]) by means of a deep tool from Diophantine approximation, namely the Schmidt Subspace Theorem (see Theorem 2.1 below). In the present note, we show how a slight modification of their proofs allows us to considerably improve two of these criteria. We begin by pointing out two important consequences of one of our new criteria. Thus, we solve two problems addressed and discussed in [1] and we establish for continued fraction expansions of algebraic numbers the analogues of the results of [3] on the expansions of algebraic numbers to an integer base.

Throughout this note, $\mathscr{C}$ denotes a finite or infinite set, called the alphabet. We identify a sequence $\mathbf{a}=\left(a_{\ell}\right)_{\ell \geq 1}$ of elements from $\mathscr{Q}$ with the infinite word $a_{1} a_{2} \ldots a_{\ell} \ldots$, as well denoted by $\mathbf{a}$. This should not cause any confusion. 
For $n \geq 1$, we denote by $p(n, \mathbf{a})$ the number of distinct blocks of $n$ consecutive letters occurring in the word $\mathbf{a}$, that is,

$$
p(n, \mathbf{a}):=\operatorname{Card}\left\{a_{\ell+1} \ldots a_{\ell+n}: \ell \geq 0\right\} .
$$

The function $n \mapsto p(n, \mathbf{a})$ is called the complexity function of $\mathbf{a}$. A well-known result of Morse and Hedlund [24, 25] asserts that $p(n, \mathbf{a}) \geq n+1$ for $n \geq 1$, unless a is ultimately periodic (in which case there exists a constant $C$ such that $p(n, \mathbf{a}) \leq C$ for $n \geq 1$ ).

Our first result asserts that the complexity function of the sequence of partial quotients $\left(a_{\ell}\right)_{\ell \geq 1}$ of an algebraic number

$$
\left[0 ; a_{1}, a_{2}, \ldots, a_{\ell}, \ldots\right]=\frac{1}{a_{1}+\frac{1}{a_{2}+\frac{1}{\ldots}}}
$$

of degree at least three cannot increase too slowly.

THEOREM 1.1. - Let $\mathbf{a}=\left(a_{\ell}\right)_{\ell \geq 1}$ be a sequence of positive integers which is not ultimately periodic. If the real number

is algebraic, then

$$
\left[0 ; a_{1}, a_{2}, \ldots, a_{\ell}, \ldots\right]
$$

Theorem 1.1 improves Theorem 7 from [12] and Theorem 4 from [1], where

$$
\lim _{n \rightarrow+\infty} p(n, \mathbf{a})-n=+\infty
$$

was proved instead of (1.1). This gives a positive answer to Problem 3 of [1] (we have chosen here a different formulation).

An infinite sequence $\mathbf{a}=\left(a_{\ell}\right)_{\ell \geq 1}$ is an automatic sequence if it can be generated by a finite automaton, that is, if there exists an integer $k \geq 2$ such that $a_{\ell}$ is a finite-state function of the representation of $\ell$ in base $k$, for every $\ell \geq 1$. We refer the reader to [13] for a more precise definition and examples of automatic sequences. Let $b \geq 2$ be an integer. In 1968, Cobham [19] asked whether a real number whose $b$-ary expansion can be generated by a finite automaton is always either rational or transcendental. A positive answer to Cobham's question was recently given in [3]. We addressed in [1] the analogous question for continued fraction expansions. Since the complexity function of every automatic sequence a satisfies $p(n, \mathbf{a})=O(n)$ (this was proved by Cobham [20] in 1972), Theorem 1.1 implies straightforwardly a negative answer to Problem 1 of [1].

THeOREм 1.2. - The continued fraction expansion of an algebraic number of degree at least three cannot be generated by a finite automaton.

The proofs of Theorems 1.1 and 1.2 rest ultimately on a combinatorial transcendence criterion established by means of the Schmidt Subspace Theorem. This is also the case for the similar results about expansions of irrational algebraic numbers to an integer base, see $[3,10]$.

$4^{\text {e }}$ SÉRIE - TOME $46-2013-$ No $^{\circ} 6$ 
Before stating our criteria, we introduce some notation. The length of a word $W$ on the alphabet $\mathscr{Q}$, that is, the number of letters composing $W$, is denoted by $|W|$. We denote the mirror image of a finite word $W:=a_{1} \ldots a_{\ell}$ by $\bar{W}:=a_{\ell} \ldots a_{1}$. In particular, $W$ is a palindrome if, and only if, $W=\bar{W}$.

Let $\mathbf{a}=\left(a_{\ell}\right)_{\ell \geq 1}$ be a sequence of elements from $\mathscr{G}$. We say that a satisfies Condition $(*)$ if a is not ultimately periodic and if there exist three sequences of finite words $\left(U_{n}\right)_{n \geq 1},\left(V_{n}\right)_{n \geq 1}$ and $\left(W_{n}\right)_{n \geq 1}$ such that:

(i) For every $n \geq 1$, either the word $W_{n} U_{n} V_{n} U_{n}$ or the word $W_{n} U_{n} V_{n} \bar{U}_{n}$ is a prefix of the word a;

(ii) The sequence $\left(\left|V_{n}\right| /\left|U_{n}\right|\right)_{n \geq 1}$ is bounded from above;

(iii) The sequence $\left(\left|W_{n}\right| /\left|U_{n}\right|\right)_{n \geq 1}$ is bounded from above;

(iv) The sequence $\left(\left|U_{n}\right|\right)_{n \geq 1}$ is increasing.

Equivalently, the word a satisfies Condition $(*)$ if there exists a positive real number $\varepsilon$ such that, for arbitrarily large integers $N$, the prefix $a_{1} a_{2} \ldots a_{N}$ of a contains two disjoint occurrences of a word of length $[\varepsilon N]$ or it contains a word $U$ of length $[\varepsilon N]$ and its mirror image $\bar{U}$, provided that $U$ and $\bar{U}$ do not overlap. Here and below, [.] denotes the integer part function.

We summarize our two new combinatorial transcendence criteria in the following theorem.

TheOrem 1.3. - Let $\mathbf{a}=\left(a_{\ell}\right)_{\ell \geq 1}$ be a sequence of positive integers. Let $\left(p_{\ell} / q_{\ell}\right)_{\ell \geq 1}$ denote the sequence of convergents to the real number

$$
\alpha:=\left[0 ; a_{1}, a_{2}, \ldots, a_{\ell}, \ldots\right] .
$$

Assume that the sequence $\left(q_{\ell}^{1 / \ell}\right)_{\ell \geq 1}$ is bounded. If $\mathbf{a}$ satisfies Condition $(*)$, then $\alpha$ is transcendental.

Theorem 1.3 is the disjoint union of two transcendence criteria. A first one applies to stammering continued fractions, where the terminology 'stammering' means that in (i) the word $W_{n} U_{n} V_{n} U_{n}$ is a prefix of the word a for infinitely many $n$; see Theorem 3.1. A second one is concerned with quasi-palindromic continued fractions, where the terminology 'quasipalindromic' means that in (i) the word $W_{n} U_{n} V_{n} \bar{U}_{n}$ is a prefix of the word a for infinitely many $n$; see Theorem 5.1. The condition that the sequence $\left(q_{\ell}^{1 / \ell}\right)_{\ell \geq 1}$ has to be bounded is not very restrictive, since it is satisfied by almost all real numbers (in the sense of the Lebesgue measure). Furthermore, it is clearly satisfied when $\left(a_{\ell}\right)_{\ell \geq 1}$ is bounded. Note that this condition can be removed if a begins with arbitrarily large squares $U_{n} U_{n}$ (Theorem 2.1 from [9]) or with arbitrarily large palindromes $U_{n} \bar{U}_{n}$ (Theorem 2.1 from [5]).

Theorem 1.3 encompasses all the combinatorial transcendence criteria for continued fraction expansions established in $[1,4,5,9]$ under the assumption that the sequence $\left(q_{\ell}^{1 / \ell}\right)_{\ell \geq 1}$ is bounded.

Let $\mathbf{a}$ be a sequence of positive integers. If there exist three sequences of finite words $\left(U_{n}\right)_{n \geq 1},\left(V_{n}\right)_{n \geq 1}$ and $\left(W_{n}\right)_{n \geq 1}$ such that $\lim \sup _{n \rightarrow+\infty}\left|W_{n}\right| /\left|U_{n}\right|$ is sufficiently small and a satisfies Condition $(*)$, then the transcendence of $\left[0 ; a_{1}, a_{2}, \ldots\right]$ was already proved in $[1,9,5]$. The novelty in Theorem 1.3 is that we allow $\left|W_{n}\right|$ to be large, provided however that 
the quotients $\left|W_{n}\right| /\left|U_{n}\right|$ remain bounded independently of $n$. This is crucial for the proofs of Theorems 1.1 and 1.2.

At present, we do not know any transcendence criterion involving palindromes for expansions to integer bases; however, see [2].

We end this section with an application of Theorem 3.1 to quasi-periodic continued fractions.

Theorem 1.4. - Consider the quasi-periodic continued fraction

$$
\alpha=[0 ; a_{1}, \ldots, a_{n_{0}-1}, \underbrace{a_{n_{0}}, \ldots, a_{n_{0}+r_{0}-1}}_{\lambda_{0}}, \underbrace{a_{n_{1}}, \ldots, a_{n_{1}+r_{1}-1}}_{\lambda_{1}}, \ldots],
$$

where the notation means that $n_{k+1}=n_{k}+\lambda_{k} r_{k}$ and the $\lambda$ 's indicate the number of times a block of partial quotients is repeated. Let $\left(p_{\ell} / q_{\ell}\right)_{\ell \geq 1}$ denote the sequence of convergents to $\alpha$. Assume that the sequence $\left(q_{\ell}^{1 / \ell}\right)_{\ell \geq 1}$ is bounded. If the sequence $\left(a_{\ell}\right)_{\ell \geq 1}$ is not ultimately periodic and

$$
\liminf _{k \rightarrow \infty} \frac{\lambda_{k+1}}{\lambda_{k}}>1
$$

then the real number $\alpha$ is transcendental.

Theorem 1.4 improves Theorem 3.4 from [4], where, instead of the Assumption (1.2), the stronger condition $\liminf _{k \rightarrow \infty} \lambda_{k+1} / \lambda_{k}>2$ was required.

\section{Auxiliary results}

We gather below several classical results from the theory of continued fractions. Standard references include [26, 22, 29].

Let $\alpha:=\left[0 ; a_{1}, a_{2}, \ldots\right]$ be a real irrational number. Set $p_{-1}=q_{0}=1$ and $q_{-1}=p_{0}=0$. For $\ell \geq 1$, the $\ell$-th convergent to $\alpha$ is the rational number $p_{\ell} / q_{\ell}:=\left[0 ; a_{1}, a_{2}, \ldots, a_{\ell}\right]$. Observe that

$$
q_{\ell}=a_{\ell} q_{\ell-1}+q_{\ell-2}, \quad \ell \geq 1 .
$$

Furthermore, the sequence $\left(q_{\ell}\right)_{\ell \geq 1}$ is increasing and $q_{\ell}$ and $q_{\ell+1}$ are coprime for $\ell \geq 0$.

The theory of continued fraction implies that (see e.g., Theorem 164 of [22])

$$
\left|q_{\ell} \alpha-p_{\ell}\right|<q_{\ell+1}^{-1}, \quad \text { for } \ell \geq 1,
$$

and

$$
q_{\ell+h} \geq q_{\ell}(\sqrt{2})^{h-1}, \quad \text { for } h, \ell \geq 1 .
$$

Indeed, an easy induction on $h$ based on (2.1) proves (2.3) for every fixed value of $\ell \geq 1$.

Likewise, an induction based on (2.1) allows us to establish the mirror formula

$$
\frac{q_{\ell-1}}{q_{\ell}}=\left[0 ; a_{\ell}, a_{\ell-1}, \ldots, a_{1}\right], \quad \ell \geq 1 .
$$

The main tool for the proof of Theorem 1.3 is the Schmidt Subspace Theorem. 
Theorem 2.1 (W. M. Schmidt). - Let $m \geq 2$ be an integer. Let $L_{1}, \ldots, L_{m}$ be linearly independent linear forms in $\mathbf{x}=\left(x_{1}, \ldots, x_{m}\right)$ with algebraic coefficients. Let $\varepsilon$ be a positive real number. Then, the set of solutions $\mathbf{x}=\left(x_{1}, \ldots, x_{m}\right)$ in $\mathbf{Z}^{m}$ to the inequality

$$
\left|L_{1}(\mathbf{x}) \ldots L_{m}(\mathbf{x})\right| \leq\left(\max \left\{\left|x_{1}\right|, \ldots,\left|x_{m}\right|\right\}\right)^{-\varepsilon}
$$

lies in finitely many proper linear subspaces of $\mathbf{Q}^{m}$.

Proof. - See e.g., [28, 29].

\section{Transcendence criterion for stammering continued fractions}

In this section we establish the part of Theorem 1.3 dealing with stammering continued fractions. Let $\mathbf{a}=\left(a_{\ell}\right)_{\ell \geq 1}$ be a sequence of elements from $\mathscr{C}$. We say that a satisfies Condition $(\boldsymbol{A})$ if $\mathbf{a}$ is not ultimately periodic and if there exist three sequences of finite words $\left(U_{n}\right)_{n \geq 1},\left(V_{n}\right)_{n \geq 1}$ and $\left(W_{n}\right)_{n \geq 1}$ such that:

(i) For every $n \geq 1$, the word $W_{n} U_{n} V_{n} U_{n}$ is a prefix of the word a;

(ii) The sequence $\left(\left|V_{n}\right| /\left|U_{n}\right|\right)_{n>1}$ is bounded from above;

(iii) The sequence $\left(\left|W_{n}\right| /\left|U_{n}\right|\right)_{n \geq 1}$ is bounded from above;

(iv) The sequence $\left(\left|U_{n}\right|\right)_{n \geq 1}$ is increasing.

THEOREM 3.1. - Let $\mathbf{a}=\left(a_{\ell}\right)_{\ell \geq 1}$ be a sequence of positive integers. Let $\left(p_{\ell} / q_{\ell}\right)_{\ell \geq 1}$ denote the sequence of convergents to the real number

$$
\alpha:=\left[0 ; a_{1}, a_{2}, \ldots, a_{\ell}, \ldots\right] .
$$

Assume that the sequence $\left(q_{\ell}^{1 / \ell}\right)_{\ell \geq 1}$ is bounded. If a satisfies Condition ( $\left.\mathbf{(}\right)$, then $\alpha$ is transcendental.

Theorem 3.1 improves Theorem 2 from [1] and Theorem 3.1 from [9]. Furthermore, it contains Theorem 3.2 from [4].

Theorem 3.1 is the exact analogue of the combinatorial transcendence criterion for expansions to integer bases proved in [10]. Although its proof is very close to that of Theorem 2 of [1], we have decided to write it completely. The new ingredient is estimate (3.4) below.

Proof. - Throughout, the constants implied in $\ll$ depend only on $\alpha$. Assume that the sequences $\left(U_{n}\right)_{n \geq 1},\left(V_{n}\right)_{n \geq 1}$ and $\left(W_{n}\right)_{n \geq 1}$ occurring in the definition of Condition $(\boldsymbol{\phi})$ are fixed. For $n \geq 1$, set $u_{n}=\left|U_{n}\right|, v_{n}=\left|V_{n}\right|$ and $w_{n}=\left|W_{n}\right|$. We assume that the real number $\alpha:=\left[0 ; a_{1}, a_{2}, \ldots\right]$ is algebraic of degree at least three. Set $p_{-1}=q_{0}=1$ and $q_{-1}=p_{0}=0$.

We observe that $\alpha$ admits infinitely many good quadratic approximants obtained by truncating its continued fraction expansion and completing by periodicity. Precisely, for every positive integer $n$, we define the sequence $\left(b_{k}^{(n)}\right)_{k \geq 1}$ by

$$
\begin{gathered}
b_{h}^{(n)}=a_{h} \quad \text { for } 1 \leq h \leq w_{n}+u_{n}+v_{n}, \\
b_{w_{n}+h+j\left(u_{n}+v_{n}\right)}^{(n)}=a_{w_{n}+h} \quad \text { for } 1 \leq h \leq u_{n}+v_{n} \text { and } j \geq 0 .
\end{gathered}
$$

The sequence $\left(b_{k}^{(n)}\right)_{k \geq 1}$ is ultimately periodic, with preperiod $W_{n}$ and with period $U_{n} V_{n}$. Set

$$
\alpha_{n}=\left[0 ; b_{1}^{(n)}, b_{2}^{(n)}, \ldots, b_{k}^{(n)}, \ldots\right]
$$


and note that, since the first $w_{n}+u_{n}+v_{n}+u_{n}$ partial quotients of $\alpha$ and of $\alpha_{n}$ are the same, it follows from (2.2) that

$$
\left|\alpha-\frac{p_{w_{n}+2 u_{n}+v_{n}}}{q_{w_{n}+2 u_{n}+v_{n}}}\right|<\frac{1}{q_{w_{n}+2 u_{n}+v_{n}}^{2}} \quad \text { and } \quad\left|\alpha_{n}-\frac{p_{w_{n}+2 u_{n}+v_{n}}}{q_{w_{n}+2 u_{n}+v_{n}}}\right|<\frac{1}{q_{w_{n}+2 u_{n}+v_{n}}^{2}},
$$

thus,

$$
\left|\alpha-\alpha_{n}\right| \leq 2 q_{w_{n}+2 u_{n}+v_{n}}^{-2} .
$$

Furthermore, an elementary computation (see e.g., [26] on page 71) shows that $\alpha_{n}$ is root of the quadratic polynomial

$$
\begin{aligned}
P_{n}(X):= & \left(q_{w_{n}-1} q_{w_{n}+u_{n}+v_{n}}-q_{w_{n}} q_{w_{n}+u_{n}+v_{n}-1}\right) X^{2} \\
& -\left(q_{w_{n}-1} p_{w_{n}+u_{n}+v_{n}}-q_{w_{n}} p_{w_{n}+u_{n}+v_{n}-1}\right. \\
& \left.+p_{w_{n}-1} q_{w_{n}+u_{n}+v_{n}}-p_{w_{n}} q_{w_{n}+u_{n}+v_{n}-1}\right) X \\
& +\left(p_{w_{n}-1} p_{w_{n}+u_{n}+v_{n}}-p_{w_{n}} p_{w_{n}+u_{n}+v_{n}-1}\right) .
\end{aligned}
$$

Since $\alpha_{n}$ lies in $(0,1)$, we have $p_{\ell} \leq q_{\ell}$ for $\ell \geq 1$ and the height $H\left(P_{n}\right)$ of the polynomial $P_{n}(X)$ (the height $H(P)$ of an integer polynomial $P(X)$ is the maximum of the absolute values of its coefficients) is at most equal to $2 q_{w_{n}} q_{w_{n}+u_{n}+v_{n}}$. By (2.2), we have

$$
\begin{aligned}
& \left|\left(q_{w_{n}-1} q_{w_{n}+u_{n}+v_{n}}-q_{w_{n}} q_{w_{n}+u_{n}+v_{n}-1}\right) \alpha-\left(q_{w_{n}-1} p_{w_{n}+u_{n}+v_{n}}-q_{w_{n}} p_{w_{n}+u_{n}+v_{n}-1}\right)\right| \\
& \quad \leq q_{w_{n}-1}\left|q_{w_{n}+u_{n}+v_{n}} \alpha-p_{w_{n}+u_{n}+v_{n}}\right|+q_{w_{n}}\left|q_{w_{n}+u_{n}+v_{n}-1} \alpha-p_{w_{n}+u_{n}+v_{n}-1}\right| \\
& \quad \leq 2 q_{w_{n}} q_{w_{n}+u_{n}+v_{n}}^{-1}
\end{aligned}
$$

and, likewise,

$$
\begin{aligned}
& \left|\left(q_{w_{n}-1} q_{w_{n}+u_{n}+v_{n}}-q_{w_{n}} q_{w_{n}+u_{n}+v_{n}-1}\right) \alpha-\left(p_{w_{n}-1} q_{w_{n}+u_{n}+v_{n}}-p_{w_{n}} q_{w_{n}+u_{n}+v_{n}-1}\right)\right| \\
& \quad \leq q_{w_{n}+u_{n}+v_{n}}\left|q_{w_{n}-1} \alpha-p_{w_{n}-1}\right|+q_{w_{n}+u_{n}+v_{n}-1}\left|q_{w_{n}} \alpha-p_{w_{n}}\right| \\
& \quad \leq 2 q_{w_{n}}^{-1} q_{w_{n}+u_{n}+v_{n}} .
\end{aligned}
$$

Using (3.1), (3.2), and (3.3), we then get

(3.4)

$$
\begin{aligned}
& \left|P_{n}(\alpha)\right|=\left|P_{n}(\alpha)-P_{n}\left(\alpha_{n}\right)\right| \\
& =\mid\left(q_{w_{n}-1} q_{w_{n}+u_{n}+v_{n}}-q_{w_{n}} q_{w_{n}+u_{n}+v_{n}-1}\right)\left(\alpha-\alpha_{n}\right)\left(\alpha+\alpha_{n}\right) \\
& -\left(q_{w_{n}-1} p_{w_{n}+u_{n}+v_{n}}-q_{w_{n}} p_{w_{n}+u_{n}+v_{n}-1}+p_{w_{n}-1} q_{w_{n}+u_{n}+v_{n}}-p_{w_{n}} q_{w_{n}+u_{n}+v_{n}-1}\right)\left(\alpha-\alpha_{n}\right) \mid \\
& =\mid\left(q_{w_{n}-1} q_{w_{n}+u_{n}+v_{n}}-q_{w_{n}} q_{w_{n}+u_{n}+v_{n}-1}\right) \alpha-\left(q_{w_{n}-1} p_{w_{n}+u_{n}+v_{n}}-q_{w_{n}} p_{w_{n}+u_{n}+v_{n}-1}\right) \\
& +\left(q_{w_{n}-1} q_{w_{n}+u_{n}+v_{n}}-q_{w_{n}} q_{w_{n}+u_{n}+v_{n}-1}\right) \alpha-\left(p_{w_{n}-1} q_{w_{n}+u_{n}+v_{n}}-p_{w_{n}} q_{w_{n}+u_{n}+v_{n}-1}\right) \\
& +\left(q_{w_{n}-1} q_{w_{n}}+u_{n}+v_{n}-q_{w_{n}} q_{w_{n}+u_{n}+v_{n}-1}\right)\left(\alpha_{n}-\alpha\right)|\cdot| \alpha-\alpha_{n} \mid \\
& \ll\left|\alpha-\alpha_{n}\right| \cdot\left(q_{w_{n}} q_{w_{n}+u_{n}+v_{n}}^{-1}+q_{w_{n}}^{-1} q_{w_{n}+u_{n}+v_{n}}+q_{w_{n}} q_{w_{n}+u_{n}+v_{n}}\left|\alpha-\alpha_{n}\right|\right) \\
& \ll\left|\alpha-\alpha_{n}\right| q_{w_{n}}^{-1} q_{w_{n}+u_{n}+v_{n}} \\
& \ll q_{w_{n}}^{-1} q_{w_{n}+u_{n}+v_{n}} q_{w_{n}+2 u_{n}+v_{n}}^{-2} .
\end{aligned}
$$

This estimate is more precise than the upper bound

$$
\left|P_{n}(\alpha)\right| \ll H\left(P_{n}\right) \cdot\left|\alpha-\alpha_{n}\right| \ll q_{w_{n}} q_{w_{n}+u_{n}+v_{n}} q_{w_{n}+2 u_{n}+v_{n}}^{-2}
$$


used in [1]; namely, we gain a factor $q_{w_{n}}^{-2}$. This improvement is crucial when $w_{n}$ is large.

We consider the four linearly independent linear forms:

$$
\begin{aligned}
& L_{1}\left(X_{1}, X_{2}, X_{3}, X_{4}\right)=\alpha^{2} X_{1}-\alpha\left(X_{2}+X_{3}\right)+X_{4}, \\
& L_{2}\left(X_{1}, X_{2}, X_{3}, X_{4}\right)=\alpha X_{1}-X_{2}, \\
& L_{3}\left(X_{1}, X_{2}, X_{3}, X_{4}\right)=\alpha X_{1}-X_{3}, \\
& L_{4}\left(X_{1}, X_{2}, X_{3}, X_{4}\right)=X_{1} .
\end{aligned}
$$

Evaluating them on the quadruple

$$
\begin{aligned}
\underline{v}_{n}:= & \left(q_{w_{n}-1} q_{w_{n}+u_{n}+v_{n}}-q_{w_{n}} q_{w_{n}+u_{n}+v_{n}-1}, q_{w_{n}-1} p_{w_{n}+u_{n}+v_{n}}-q_{w_{n}} p_{w_{n}+u_{n}+v_{n}-1},\right. \\
& \left.p_{w_{n}-1} q_{w_{n}+u_{n}+v_{n}}-p_{w_{n}} q_{w_{n}+u_{n}+v_{n}-1}, p_{w_{n}-1} p_{w_{n}+u_{n}+v_{n}}-p_{w_{n}} p_{w_{n}+u_{n}+v_{n}-1}\right),
\end{aligned}
$$

it follows from (3.2), (3.3), (3.4), and (2.3) that

$$
\begin{aligned}
\prod_{1 \leq j \leq 4}\left|L_{j}\left(\underline{v}_{n}\right)\right| & \ll q_{w_{n}+u_{n}+v_{n}}^{2} q_{w_{n}+2 u_{n}+v_{n}}^{-2} \\
& \ll 2^{-u_{n}} \\
& \ll\left(q_{w_{n}} q_{w_{n}+u_{n}+v_{n}}\right)^{-\delta u_{n} /\left(2 w_{n}+u_{n}+v_{n}\right)},
\end{aligned}
$$

if $n$ is sufficiently large, where we have set

$$
M=1+\limsup _{\ell \rightarrow+\infty} q_{\ell}^{1 / \ell} \quad \text { and } \quad \delta=\frac{\log 2}{\log M} .
$$

Since a satisfies Condition (\$), we have

$$
\liminf _{n \rightarrow+\infty} \frac{u_{n}}{2 w_{n}+u_{n}+v_{n}}>0
$$

Consequently, there exists $\varepsilon>0$ such that

$$
\prod_{1 \leq j \leq 4}\left|L_{j}\left(\underline{v}_{n}\right)\right| \ll\left(q_{w_{n}} q_{w_{n}+u_{n}+v_{n}}\right)^{-\varepsilon}
$$

holds for any sufficiently large integer $n$.

It then follows from Theorem 2.1 that the points $\underline{v}_{n}$ lie in a finite union of proper linear subspaces of $\mathbf{Q}^{4}$. Thus, there exist a non-zero integer quadruple $\left(x_{1}, x_{2}, x_{3}, x_{4}\right)$ and an infinite set $\mathcal{N}_{1}$ of distinct positive integers such that

$$
\begin{aligned}
& x_{1}\left(q_{w_{n}-1} q_{w_{n}+u_{n}+v_{n}}-q_{w_{n}} q_{w_{n}+u_{n}+v_{n}-1}\right)+x_{2}\left(q_{w_{n}-1} p_{w_{n}+u_{n}+v_{n}}-q_{w_{n}} p_{w_{n}+u_{n}+v_{n}-1}\right) \\
& +x_{3}\left(p_{w_{n}-1} q_{w_{n}+u_{n}+v_{n}}-p_{w_{n}} q_{w_{n}+u_{n}+v_{n}-1}\right)+x_{4}\left(p_{w_{n}-1} p_{w_{n}+u_{n}+v_{n}}-p_{w_{n}} p_{w_{n}+u_{n}+v_{n}-1}\right) \\
& \quad=0
\end{aligned}
$$

for any $n$ in $\mathcal{N}_{1}$.

- First case: we assume that there exist an integer $\ell$ and infinitely many integers $n$ in $\mathcal{N}_{1}$ with $w_{n}=\ell$.

By extracting an infinite subset of $\mathcal{N}_{1}$ if necessary and by considering the real number $\left[0 ; a_{\ell+1}, a_{\ell+2}, \ldots\right]$ instead of $\alpha$, we may without loss of generality assume that $w_{n}=\ell=0$ for any $n$ in $\mathcal{N}_{1}$. 
Then, recalling that $q_{-1}=p_{0}=0$ and $q_{0}=p_{-1}=1$, we deduce from (3.5) that

$$
x_{1} q_{u_{n}+v_{n}-1}+x_{2} p_{u_{n}+v_{n}-1}-x_{3} q_{u_{n}+v_{n}}-x_{4} p_{u_{n}+v_{n}}=0,
$$

for any $n$ in $\mathcal{N}_{1}$. Observe that $\left(x_{1}, x_{2}\right) \neq(0,0)$, since, otherwise, by letting $n$ tend to infinity along $\mathcal{N}_{1}$ in (3.6), we would get that the real number $\alpha$ is rational. Dividing (3.6) by $q_{u_{n}+v_{n}}$, we obtain

$$
x_{1} \frac{q_{u_{n}+v_{n}-1}}{q_{u_{n}+v_{n}}}+x_{2} \frac{p_{u_{n}+v_{n}-1}}{q_{u_{n}+v_{n}-1}} \cdot \frac{q_{u_{n}+v_{n}-1}}{q_{u_{n}+v_{n}}}-x_{3}-x_{4} \frac{p_{u_{n}+v_{n}}}{q_{u_{n}+v_{n}}}=0 .
$$

By letting $n$ tend to infinity along $\mathcal{N}_{1}$ in (3.7), we get that

$$
\beta:=\lim _{\mathcal{N}_{1} \ni n \rightarrow+\infty} \frac{q_{u_{n}+v_{n}-1}}{q_{u_{n}+v_{n}}}=\frac{x_{3}+x_{4} \alpha}{x_{1}+x_{2} \alpha} .
$$

Furthermore, observe that, for any sufficiently large integer $n$ in $\mathcal{N}_{1}$, we have

$$
\left|\beta-\frac{q_{u_{n}+v_{n}-1}}{q_{u_{n}+v_{n}}}\right|=\left|\frac{x_{3}+x_{4} \alpha}{x_{1}+x_{2} \alpha}-\frac{x_{3}+x_{4} p_{u_{n}+v_{n}} / q_{u_{n}+v_{n}}}{x_{1}+x_{2} p_{u_{n}+v_{n}-1} / q_{u_{n}+v_{n}-1}}\right| \ll \frac{1}{q_{u_{n}+v_{n}-1} q_{u_{n}+v_{n}}},
$$

by (2.2). Since the rational number $q_{u_{n}+v_{n}-1} / q_{u_{n}+v_{n}}$ is under its reduced form and $u_{n}+v_{n}$ tends to infinity when $n$ tends to infinity along $\mathcal{N}_{1}$, we see that, for every positive real number $\eta$ and every positive integer $N$, there exists a reduced rational number $a / b$ such that $b>N$ and $|\beta-a / b| \leq \eta / b$. This implies that $\beta$ is irrational.

Consider now the three linearly independent linear forms

$$
L_{1}^{\prime}\left(Y_{1}, Y_{2}, Y_{3}\right)=\beta Y_{1}-Y_{2}, \quad L_{2}^{\prime}\left(Y_{1}, Y_{2}, Y_{3}\right)=\alpha Y_{1}-Y_{3}, \quad L_{3}^{\prime}\left(Y_{1}, Y_{2}, Y_{3}\right)=Y_{2} .
$$

Evaluating them on the triple $\left(q_{u_{n}+v_{n}}, q_{u_{n}+v_{n}-1}, p_{u_{n}+v_{n}}\right)$ with $n \in \mathcal{N}_{1}$, we infer from (2.2) and (3.8) that

$$
\prod_{1 \leq j \leq 3}\left|L_{j}^{\prime}\left(q_{u_{n}+v_{n}}, q_{u_{n}+v_{n}-1}, p_{u_{n}+v_{n}}\right)\right| \ll q_{u_{n}+v_{n}}^{-1} .
$$

It then follows from Theorem 2.1 that the points $\left(q_{u_{n}+v_{n}}, q_{u_{n}+v_{n}-1}, p_{u_{n}+v_{n}}\right)$ with $n \in \mathcal{N}_{1}$ lie in a finite union of proper linear subspaces of $\mathbf{Q}^{3}$. Thus, there exist a non-zero integer triple $\left(y_{1}, y_{2}, y_{3}\right)$ and an infinite set of distinct positive integers $\mathcal{N}_{2} \subset \mathcal{N}_{1}$ such that

$$
y_{1} q_{u_{n}+v_{n}}+y_{2} q_{u_{n}+v_{n}-1}+y_{3} p_{u_{n}+v_{n}}=0
$$

for any $n$ in $\mathcal{N}_{2}$. Dividing (3.9) by $q_{u_{n}+v_{n}}$ and letting $n$ tend to infinity along $\mathcal{N}_{2}$, we get

$$
y_{1}+y_{2} \beta+y_{3} \alpha=0 \text {. }
$$

To obtain another equation linking $\alpha$ and $\beta$, we consider the three linearly independent linear forms

$$
L_{1}^{\prime \prime}\left(Z_{1}, Z_{2}, Z_{3}\right)=\beta Z_{1}-Z_{2}, \quad L_{2}^{\prime \prime}\left(Z_{1}, Z_{2}, Z_{3}\right)=\alpha Z_{2}-Z_{3}, \quad L_{3}^{\prime \prime}\left(Z_{1}, Z_{2}, Z_{3}\right)=Z_{2} .
$$

Evaluating them on the triple $\left(q_{u_{n}+v_{n}}, q_{u_{n}+v_{n}-1}, p_{u_{n}+v_{n}-1}\right)$ with $n$ in $\mathcal{N}_{1}$, we infer from (2.2) and (3.8) that

$$
\prod_{1 \leq j \leq 3}\left|L_{j}^{\prime \prime}\left(q_{u_{n}+v_{n}}, q_{u_{n}+v_{n}-1}, p_{u_{n}+v_{n}-1}\right)\right| \ll q_{u_{n}+v_{n}}^{-1} .
$$

$4^{\text {e }}$ SÉRIE - TOME $46-2013$ - No 6 
It then follows from Theorem 2.1 that the points $\left(q_{u_{n}+v_{n}}, q_{u_{n}+v_{n}-1}, p_{u_{n}+v_{n}-1}\right)$ with $n \in \mathcal{N}_{1}$ lie in a finite union of proper linear subspaces of $\mathbf{Q}^{3}$. Thus, there exist a non-zero integer triple $\left(z_{1}, z_{2}, z_{3}\right)$ and an infinite set of distinct positive integers $\mathcal{N}_{3} \subset \mathcal{N}_{2}$ such that

$$
z_{1} q_{u_{n}+v_{n}}+z_{2} q_{u_{n}+v_{n}-1}+z_{3} p_{u_{n}+v_{n}-1}=0,
$$

for any $n$ in $\mathcal{N}_{3}$. Dividing (3.11) by $q_{u_{n}+v_{n}-1}$ and letting $n$ tend to infinity along $\mathcal{N}_{3}$, we get

$$
\frac{z_{1}}{\beta}+z_{2}+z_{3} \alpha=0
$$

We infer from (3.10) and (3.12) that

$$
\left(z_{3} \alpha+z_{2}\right)\left(y_{3} \alpha+y_{1}\right)=y_{2} z_{1} .
$$

Since $\beta$ is irrational, we get from (3.10) and (3.12) that $y_{3} z_{3} \neq 0$. This shows that $\alpha$ is an algebraic number of degree at most two, which is a contradiction with our assumption that $\alpha$ is algebraic of degree at least three.

- Second case: extracting an infinite subset $\mathcal{N}_{4}$ of $\mathcal{N}_{1}$ if necessary, we assume that $\left(w_{n}\right)_{n \in \mathcal{N}_{4}}$ tends to infinity.

In particular $\left(p_{w_{n}} / q_{w_{n}}\right)_{n \in \mathcal{N}_{4}}$ and $\left(p_{w_{n}+u_{n}+v_{n}} / q_{w_{n}+u_{n}+v_{n}}\right)_{n \in \mathcal{N}_{4}}$ both tend to $\alpha$ as $n$ tends to infinity.

We make the following observation. Let $a$ be a letter and $U, V, W$ be three finite words ( $V$ may be empty) such that a begins with $W U V U$ and $a$ is the last letter of $W$ and of $U V$. Then, writing $W=W^{\prime} a, V=V^{\prime} a$ if $V$ is non-empty, and $U=U^{\prime} a$ if $V$ is empty, we see that a begins with $W^{\prime}(a U) V^{\prime}(a U)$ if $V$ is non-empty and with $W^{\prime}\left(a U^{\prime}\right)\left(a U^{\prime}\right)$ if $V$ is empty. Consequently, by iterating this remark if necessary, we can assume that for any $n$ in $\mathcal{N}_{4}$, the last letter of the word $U_{n} V_{n}$ differs from the last letter of the word $W_{n}$. Said differently, we have $a_{w_{n}} \neq a_{w_{n}+u_{n}+v_{n}}$ for any $n$ in $\mathcal{N}_{4}$.

Divide (3.5) by $q_{w_{n}} q_{w_{n}+u_{n}+v_{n}-1}$ and write

$$
Q_{n}:=\left(q_{w_{n}-1} q_{w_{n}+u_{n}+v_{n}}\right) /\left(q_{w_{n}} q_{w_{n}+u_{n}+v_{n}-1}\right) .
$$

We then get

$$
\begin{aligned}
& x_{1}\left(Q_{n}-1\right)+x_{2}\left(Q_{n} \frac{p_{w_{n}+u_{n}+v_{n}}}{q_{w_{n}+u_{n}+v_{n}}}-\frac{p_{w_{n}+u_{n}+v_{n}-1}}{q_{w_{n}+u_{n}+v_{n}-1}}\right)+x_{3}\left(Q_{n} \frac{p_{w_{n}-1}}{q_{w_{n}-1}}-\frac{p_{w_{n}}}{q_{w_{n}}}\right) \\
& \quad+x_{4}\left(Q_{n} \frac{p_{w_{n}-1}}{q_{w_{n}-1}} \frac{p_{w_{n}+u_{n}+v_{n}}}{q_{w_{n}+u_{n}+v_{n}}}-\frac{p_{w_{n}}}{q_{w_{n}}} \frac{p_{w_{n}+u_{n}+v_{n}-1}}{q_{w_{n}+u_{n}+v_{n}-1}}\right)=0,
\end{aligned}
$$

for any $n$ in $\mathcal{N}_{4}$. To shorten the notation, for any $\ell \geq 1$, we put $R_{\ell}:=\alpha-p_{\ell} / q_{\ell}$ and rewrite (3.13) as

$$
\begin{aligned}
& x_{1}\left(Q_{n}-1\right)+x_{2}\left(Q_{n}\left(\alpha-R_{w_{n}+u_{n}+v_{n}}\right)-\left(\alpha-R_{w_{n}+u_{n}+v_{n}-1}\right)\right) \\
& \quad+x_{3}\left(Q_{n}\left(\alpha-R_{w_{n}-1}\right)-\left(\alpha-R_{w_{n}}\right)\right) \\
& \quad+x_{4}\left(Q_{n}\left(\alpha-R_{w_{n}-1}\right)\left(\alpha-R_{w_{n}+u_{n}+v_{n}}\right)-\left(\alpha-R_{w_{n}}\right)\left(\alpha-R_{w_{n}+u_{n}+v_{n}-1}\right)\right)=0 .
\end{aligned}
$$


This yields

$$
\begin{aligned}
\left(Q_{n}-1\right)( & \left.x_{1}+\left(x_{2}+x_{3}\right) \alpha+x_{4} \alpha^{2}\right) \\
= & x_{2} Q_{n} R_{w_{n}+u_{n}+v_{n}}-x_{2} R_{w_{n}+u_{n}+v_{n}-1}+x_{3} Q_{n} R_{w_{n}-1}-x_{3} R_{w_{n}} \\
& -x_{4} Q_{n} R_{w_{n}-1} R_{w_{n}+u_{n}+v_{n}}+x_{4} R_{w_{n}} R_{w_{n}+u_{n}+v_{n}-1} \\
& +\alpha\left(x_{4} Q_{n} R_{w_{n}-1}+x_{4} Q_{n} R_{w_{n}+u_{n}+v_{n}}-x_{4} R_{w_{n}}-x_{4} R_{w_{n}+u_{n}+v_{n}-1}\right)
\end{aligned}
$$

Observe that by (2.2) we have

$$
\left|R_{\ell}\right| \leq q_{\ell}^{-1} q_{\ell+1}^{-1}, \quad \ell \geq 1 .
$$

We use (3.14), (3.15) and the assumption that $a_{w_{n}} \neq a_{w_{n}+u_{n}+v_{n}}$ for any $n$ in $\mathcal{N}_{4}$ to establish the following claim.

Claim. - We have

$$
x_{1}+\left(x_{2}+x_{3}\right) \alpha+x_{4} \alpha^{2}=0 .
$$

Proof of the claim. - If there are arbitrarily large integers $n$ in $\mathcal{N}_{4}$ such that $Q_{n} \geq 2$ or $Q_{n} \leq 1 / 2$, then the claim follows from (3.14) and (3.15).

Assume that $1 / 2 \leq Q_{n} \leq 2$ holds for every large $n$ in $\mathcal{N}_{4}$. We then derive from (3.14) and (3.15) that

$$
\left|\left(Q_{n}-1\right)\left(x_{1}+\left(x_{2}+x_{3}\right) \alpha+x_{4} \alpha^{2}\right)\right| \ll\left|R_{w_{n}-1}\right| \ll q_{w_{n}-1}^{-1} q_{w_{n}}^{-1} .
$$

If $x_{1}+\left(x_{2}+x_{3}\right) \alpha+x_{4} \alpha^{2} \neq 0$, then we get

$$
\left|Q_{n}-1\right| \ll q_{w_{n}-1}^{-1} q_{w_{n}}^{-1} .
$$

On the other hand, observe that, by (2.4), the rational number $Q_{n}$ is the quotient of the two continued fractions $\left[a_{w_{n}+u_{n}+v_{n}} ; a_{w_{n}+u_{n}+v_{n}-1}, \ldots, a_{1}\right]$ and $\left[a_{w_{n}} ; a_{w_{n}-1}, \ldots, a_{1}\right]$. Since $a_{w_{n}+u_{n}+v_{n}} \neq a_{w_{n}}$, we have either $a_{w_{n}+u_{n}+v_{n}}-a_{w_{n}} \geq 1$ or $a_{w_{n}}-a_{w_{n}+u_{n}+v_{n}} \geq 1$. In the former case, we see that

$$
Q_{n} \geq \frac{a_{w_{n}+u_{n}+v_{n}}}{a_{w_{n}}+\frac{1}{1+\frac{1}{a_{w_{n}-2}+1}}} \geq \frac{a_{w_{n}}+1}{a_{w_{n}}+\frac{a_{w_{n}-2}+1}{a_{w_{n}-2}+2}} \geq 1+\frac{1}{\left(a_{w_{n}}+1\right)\left(a_{w_{n}-2}+2\right)} .
$$

In the latter case, we have

$$
\frac{1}{Q_{n}} \geq \frac{a_{w_{n}}+\frac{1}{a_{w_{n}-1}+1}}{a_{w_{n}+u_{n}+v_{n}}+1} \geq 1+\frac{1}{\left(a_{w_{n}-1}+1\right)\left(a_{w_{n}+u_{n}+v_{n}}+1\right)} \geq 1+\frac{1}{\left(a_{w_{n}-1}+1\right) a_{w_{n}}} .
$$

Consequently, in any case, we have

$$
\left|Q_{n}-1\right| \gg a_{w_{n}}^{-1} \min \left\{a_{w_{n}-2}^{-1}, a_{w_{n}-1}^{-1}\right\} \gg a_{w_{n}}^{-1} q_{w_{n}-1}^{-1} .
$$

Combined with (3.16), this gives

$$
a_{w_{n}} \gg q_{w_{n}} \gg a_{w_{n}} q_{w_{n}-1},
$$

which implies that $n$ is bounded, a contradiction. This proves the claim.

$4{ }^{\text {e }}$ SÉRIE - TOME $46-2013$ - No 6 
Since $\alpha$ is irrational and not quadratic, we deduce from the claim that $x_{1}=x_{4}=0$ and $x_{2}=-x_{3}$. Then, $x_{2}$ is non-zero and, by (3.5), we have, for any $n$ in $\mathcal{N}_{4}$,

$$
q_{w_{n}-1} p_{w_{n}+u_{n}+v_{n}}-q_{w_{n}} p_{w_{n}+u_{n}+v_{n}-1}=p_{w_{n}-1} q_{w_{n}+u_{n}+v_{n}}-p_{w_{n}} q_{w_{n}+u_{n}+v_{n}-1} .
$$

Thus, the polynomial $P_{n}(X)$ can simply be expressed as

$$
\begin{aligned}
P_{n}(X):= & \left(q_{w_{n}-1} q_{w_{n}+u_{n}+v_{n}}-q_{w_{n}} q_{w_{n}+u_{n}+v_{n}-1}\right) X^{2} \\
& -2\left(q_{w_{n}-1} p_{w_{n}+u_{n}+v_{n}}-q_{w_{n}} p_{w_{n}+u_{n}+v_{n}-1}\right) X \\
& +\left(p_{w_{n}-1} p_{w_{n}+u_{n}+v_{n}}-p_{w_{n}} p_{w_{n}+u_{n}+v_{n}-1}\right) .
\end{aligned}
$$

Consider now the three linearly independent linear forms

$$
\begin{aligned}
& L_{1}^{\prime \prime \prime}\left(T_{1}, T_{2}, T_{3}\right)=\alpha^{2} T_{1}-2 \alpha T_{2}+T_{3}, \\
& L_{2}^{\prime \prime \prime}\left(T_{1}, T_{2}, T_{3}\right)=\alpha T_{1}-T_{2}, \\
& L_{3}^{\prime \prime \prime}\left(T_{1}, T_{2}, T_{3}\right)=T_{1} .
\end{aligned}
$$

Evaluating them on the triple

$$
\begin{aligned}
\underline{v}_{n}^{\prime}:= & \left(q_{w_{n}-1} q_{w_{n}+u_{n}+v_{n}}-q_{w_{n}} q_{w_{n}+u_{n}+v_{n}-1},\right. \\
& q_{w_{n}-1} p_{w_{n}+u_{n}+v_{n}}-q_{w_{n}} p_{w_{n}+u_{n}+v_{n}-1}, \\
& \left.p_{w_{n}-1} p_{w_{n}+u_{n}+v_{n}}-p_{w_{n}} p_{w_{n}+u_{n}+v_{n}-1}\right),
\end{aligned}
$$

for $n$ in $\mathcal{N}_{4}$, it follows from (3.2) and (3.4) that

$$
\prod_{1 \leq j \leq 3}\left|L_{j}^{\prime \prime \prime}\left(\underline{v}_{n}^{\prime}\right)\right| \ll q_{w_{n}} q_{w_{n}+u_{n}+v_{n}} q_{w_{n}+2 u_{n}+v_{n}}^{-2} \ll\left(q_{w_{n}} q_{w_{n}+u_{n}+v_{n}}\right)^{-\varepsilon},
$$

with the same $\varepsilon$ as above, if $n$ is sufficiently large.

We then deduce from Theorem 2.1 that the points $\underline{v}_{n}^{\prime}, n \in \mathcal{N}_{4}$, lie in a finite union of proper linear subspaces of $\mathbf{Q}^{3}$. Thus, there exist a non-zero integer triple $\left(t_{1}, t_{2}, t_{3}\right)$ and an infinite set of distinct positive integers $\mathcal{N}_{5}$ included in $\mathcal{N}_{4}$ such that

$$
\begin{aligned}
& t_{1}\left(q_{w_{n}-1} q_{w_{n}+u_{n}+v_{n}}-q_{w_{n}} q_{w_{n}+u_{n}+v_{n}-1}\right) \\
& \quad+t_{2}\left(q_{w_{n}-1} p_{w_{n}+u_{n}+v_{n}}-q_{w_{n}} p_{w_{n}+u_{n}+v_{n}-1}\right) \\
& \quad+t_{3}\left(p_{w_{n}-1} p_{w_{n}+u_{n}+v_{n}}-p_{w_{n}} p_{w_{n}+u_{n}+v_{n}-1}\right)=0
\end{aligned}
$$

for any $n$ in $\mathcal{N}_{5}$.

We proceed exactly as above. Divide (3.17) by $q_{w_{n}} q_{w_{n}+u_{n}+v_{n}-1}$ and set

$$
Q_{n}:=\left(q_{w_{n}-1} q_{w_{n}+u_{n}+v_{n}}\right) /\left(q_{w_{n}} q_{w_{n}+u_{n}+v_{n}-1}\right) .
$$

We then get

$$
\begin{aligned}
t_{1}\left(Q_{n}-1\right) & +t_{2}\left(Q_{n} \frac{p_{w_{n}+u_{n}+v_{n}}}{q_{w_{n}+u_{n}+v_{n}}}-\frac{p_{w_{n}+u_{n}+v_{n}-1}}{q_{w_{n}+u_{n}+v_{n}-1}}\right) \\
& +t_{3}\left(Q_{n} \frac{p_{w_{n}-1}}{q_{w_{n}-1}} \frac{p_{w_{n}+u_{n}+v_{n}}}{q_{w_{n}+u_{n}+v_{n}}}-\frac{p_{w_{n}}}{q_{w_{n}}} \frac{p_{w_{n}+u_{n}+v_{n}-1}}{q_{w_{n}+u_{n}+v_{n}-1}}\right)=0,
\end{aligned}
$$

for any $n$ in $\mathcal{N}_{5}$. We argue as after (3.13). Since $p_{w_{n}} / q_{w_{n}}$ and $p_{w_{n}+u_{n}+v_{n}} / q_{w_{n}+u_{n}+v_{n}}$ tend to $\alpha$ as $n$ tends to infinity along $\mathcal{N}_{5}$, we derive from (3.18) that

$$
t_{1}+t_{2} \alpha+t_{3} \alpha^{2}=0,
$$


a contradiction since $\alpha$ is irrational and not quadratic. Consequently, $\alpha$ must be transcendental. This concludes the proof of the theorem.

\section{Proofs of Theorems 1.1 and 1.4}

Proof of Theorem 1.1. - Let $\mathbf{a}=a_{1} a_{2} \ldots$ be an infinite word on the alphabet $\mathbf{Z}_{\geq 1}$. Assume that (1.1) does not hold. Then, there exist an integer $C \geq 2$ and an infinite set $\mathcal{N}$ of positive integers such that

$$
p(n, \mathbf{a}) \leq C n, \quad \text { for every } n \text { in } \mathcal{N} .
$$

This implies in particular that $\mathbf{a}$ is written over a finite alphabet, thus, by (2.1), the sequence $\left(q_{\ell}^{1 / \ell}\right)_{\ell \geq 1}$ is bounded.

Let $n$ be in $\mathcal{N}$. By (4.1) and the Schubfachprinzip, there exists (at least) one block $X_{n}$ of length $n$ having (at least) two occurrences in the prefix of length $(C+1) n$ of a. Thus, there are words $W_{n}, W_{n}^{\prime}, B_{n}$ and $B_{n}^{\prime}$ such that $\left|W_{n}\right|<\left|W_{n}^{\prime}\right|$ and

$$
a_{1} \ldots a_{(C+1) n}=W_{n} X_{n} B_{n}=W_{n}^{\prime} X_{n} B_{n}^{\prime} .
$$

If $\left|W_{n} X_{n}\right| \leq\left|W_{n}^{\prime}\right|$, then define $V_{n}$ by the equality $W_{n} X_{n} V_{n}=W_{n}^{\prime}$. Observe that

$$
a_{1} \ldots a_{(C+1) n}=W_{n} X_{n} V_{n} X_{n} B_{n}^{\prime}
$$

and

$$
\frac{\left|V_{n}\right|+\left|W_{n}\right|}{\left|X_{n}\right|} \leq C
$$

Set $U_{n}:=X_{n}$.

If $\left|W_{n}^{\prime}\right|<\left|W_{n} X_{n}\right|$, then, recalling that $\left|W_{n}\right|<\left|W_{n}^{\prime}\right|$, we define $X_{n}^{\prime}$ by $W_{n}^{\prime}=W_{n} X_{n}^{\prime}$. Since $X_{n} B_{n}=X_{n}^{\prime} X_{n} B_{n}^{\prime}$ and $\left|X_{n}^{\prime}\right|<\left|X_{n}\right|$, the word $X_{n}^{\prime}$ is a strict prefix of $X_{n}$ and $X_{n}$ is a rational power of $X_{n}^{\prime}$. Thus, there are a positive integer $x_{n}$ and a rational number $y_{n}$ such that $0 \leq y_{n}<2$ and

$$
X_{n}^{\prime} X_{n}=X_{n}^{\prime 1+\left|X_{n}\right| /\left|X_{n}^{\prime}\right|}={X_{n}^{\prime}}^{2 x_{n}+y_{n}}=\left(X_{n}^{\prime x_{n}}\right)^{2} X_{n}^{\prime y_{n}} .
$$

Here and below, for a positive integer $k$, we write $Z^{k}$ for the word $Z \ldots Z$ ( $k$ times repeated concatenation of the word $Z$ ). More generally, for any positive rational number $r$ such that $r|Z|$ is an integer, we denote by $Z^{r}$ the word $Z^{[r]} Z^{\prime}$, where $Z^{\prime}$ is the prefix of $Z$ of length $(r-[r])|Z|$.

Observe that

$$
2 x_{n}\left|X_{n}^{\prime}\right|+2\left|X_{n}^{\prime}\right| \geq\left|X_{n}^{\prime} X_{n}\right|
$$

thus

$$
n=\left|X_{n}\right| \leq\left(2 x_{n}+1\right)\left|X_{n}^{\prime}\right| \leq 3 x_{n}\left|X_{n}^{\prime}\right| \text {. }
$$

Consequently, $W_{n}\left(X_{n}^{\prime x_{n}}\right)^{2}$ is a prefix of a such that

$$
\left|X_{n}^{\prime x_{n}}\right| \geq n / 3
$$

and

$$
\frac{\left|W_{n}\right|}{\left|X_{n}^{\prime x_{n}}\right|} \leq \frac{3}{n} \cdot\left((C+1) n-2\left|X_{n}^{\prime x_{n}}\right|\right) \leq 3 C+1 .
$$

Set $U_{n}:=X_{n}^{\prime x_{n}}$ and let $V_{n}$ be the empty word.

4 ${ }^{\text {e }}$ SÉRIE - TOME $46-2013$ - No 6 
It then follows from (4.2), (4.3), and (4.4) that, for every $n$ in the infinite set $\mathcal{N}$,

$$
W_{n} U_{n} V_{n} U_{n} \text { is a prefix of } \mathbf{a}
$$

with

$$
\left|W_{n}\right|+\left|V_{n}\right| \leq(3 C+1)\left|U_{n}\right| .
$$

This shows that a satisfies Condition (\$). Applying Theorem 3.1, we get that the real number $\left[0 ; a_{1}, a_{2}, \ldots\right]$ is transcendental. This proves the theorem.

Proof of Theorem 1.4. - If the sequence $\left(r_{k}\right)_{k \geq 0}$ is bounded, then Theorem 1.4 is Corollary 3.3 of [4]. Thus, we assume that $\left(r_{k}\right)_{k \geq 0}$ is unbounded and we consider the infinite set $\mathcal{K}$ composed of the positive integers $k$ such that $r_{k}>\max \left\{r_{0}, \ldots, r_{k-1}\right\}$. By the Assumption (1.2), there exist $\varepsilon>0$ and $k_{0}$ such that $\lambda_{k_{0}}>2$ and $\lambda_{k+1}>(1+\varepsilon) \lambda_{k}$ for $k \geq k_{0}$. Let $k$ be in $\mathcal{K}$ with $k>k_{0}$. Set

$$
W_{k}=a_{1} a_{2} \ldots a_{n_{k}-1}
$$

and

$$
U_{k}=\left(a_{n_{k}} \ldots a_{n_{k}+r_{k}-1}\right)^{\left[\lambda_{k} / 2\right]} .
$$

Observe that a begins with $W_{k} U_{k}^{2}$. Furthermore, setting

$$
n_{0}^{\prime}=n_{0}+\sum_{h=0}^{k_{0}-1} \lambda_{h} r_{h},
$$

we have

$$
\begin{aligned}
\left|W_{k}\right| & \leq n_{0}^{\prime}+\sum_{h=k_{0}}^{k-1} \lambda_{h} r_{h} \\
& \leq n_{0}^{\prime}+r_{k} \lambda_{k}\left(\frac{1}{1+\varepsilon}+\cdots+\frac{1}{(1+\varepsilon)^{k-k_{0}}}\right) \\
& \leq n_{0}^{\prime}+r_{k} \lambda_{k} / \varepsilon \leq 2 r_{k} \lambda_{k} / \varepsilon
\end{aligned}
$$

and

$$
\left|U_{k}\right| \geq \frac{\left(\lambda_{k}-1\right) r_{k}}{2} \geq \frac{\lambda_{k} r_{k}}{4} \geq \frac{\varepsilon}{8}\left|W_{k}\right|,
$$

for every sufficiently large $k$ in $\mathcal{K}$. Consequently, the word $\mathbf{a}=a_{1} a_{2} \ldots$ satisfies Condition (\$). We conclude by applying Theorem 3.1.

\section{Transcendence criterion for quasi-palindromic continued fractions}

In this section, we establish the part of Theorem 1.3 dealing with quasi-palindromic continued fractions. Let $\mathbf{a}=\left(a_{\ell}\right)_{\ell \geq 1}$ be a sequence of elements from $\mathscr{G}$. We say that a satisfies Condition (\$) if a is not ultimately periodic and if there exist three sequences of finite words $\left(U_{n}\right)_{n \geq 1},\left(V_{n}\right)_{n \geq 1}$ and $\left(W_{n}\right)_{n \geq 1}$ such that:

(i) For every $n \geq 1$, the word $W_{n} U_{n} V_{n} \bar{U}_{n}$ is a prefix of the word $\mathbf{a}$;

(ii) The sequence $\left(\left|V_{n}\right| /\left|U_{n}\right|\right)_{n \geq 1}$ is bounded from above;

(iii) The sequence $\left(\left|W_{n}\right| /\left|U_{n}\right|\right)_{n \geq 1}$ is bounded from above;

(iv) The sequence $\left(\left|U_{n}\right|\right)_{n \geq 1}$ is increasing. 
Theorem 5.1. - Let $\mathbf{a}=\left(a_{\ell}\right)_{\ell \geq 1}$ be a sequence of positive integers. Let $\left(p_{\ell} / q_{\ell}\right)_{\ell \geq 1}$ denote the sequence of convergents to the real number

$$
\alpha:=\left[0 ; a_{1}, a_{2}, \ldots, a_{\ell}, \ldots\right] .
$$

Assume that the sequence $\left(q_{\ell}^{1 / \ell}\right)_{\ell \geq 1}$ is bounded. If a satisfies Condition (\$), then $\alpha$ is transcendental.

Theorem 5.1 improves Theorem 2.4 from [5].

Proof. - Throughout, the constants implied in « are absolute. We content ourselves to explain which changes should be made to the proof of Theorem 2.4 from [5] in order to establish Theorem 5.1. We keep the notation of that paper.

Assume that the sequences $\left(U_{n}\right)_{n \geq 1},\left(V_{n}\right)_{n \geq 1}$ and $\left(W_{n}\right)_{n \geq 1}$ are fixed. Set $r_{n}=\left|W_{n}\right|$, $s_{n}=\left|W_{n} U_{n}\right|$ and $t_{n}=\left|W_{n} U_{n} V_{n} \overline{U_{n}}\right|$, for $n \geq 1$. Assume that the real number $\alpha:=\left[0 ; a_{1}, a_{2}, \ldots\right]$ is algebraic of degree at least three.

For $n \geq 1$, consider the rational number $P_{n} / Q_{n}$ defined by

$$
\frac{P_{n}}{Q_{n}}:=\left[0 ; W_{n} U_{n} V_{n} \overline{U_{n}} \overline{W_{n}}\right]
$$

and denote by $P_{n}^{\prime} / Q_{n}^{\prime}$ the last convergent to $P_{n} / Q_{n}$ which is different from $P_{n} / Q_{n}$. It has been proved in [5] that

$$
\begin{gathered}
\left|Q_{n} \alpha-P_{n}\right|<Q_{n} q_{t_{n}}^{-2}, \quad\left|Q_{n}^{\prime} \alpha-P_{n}^{\prime}\right|<Q_{n} q_{t_{n}}^{-2}, \\
\left|Q_{n} \alpha-Q_{n}^{\prime}\right|<Q_{n} q_{s_{n}}^{-2},
\end{gathered}
$$

and

$$
Q_{n} \leq 2 q_{r_{n}} q_{t_{n}} \leq 2 q_{s_{n}} q_{t_{n}} .
$$

Inequality (5.2) is a consequence of the mirror formula (2.4) which is a key ingredient for the proof of the combinatorial transcendence criteria for quasi-palindromic continued fractions. Since

$$
\begin{aligned}
\alpha\left(Q_{n} \alpha-P_{n}\right)-\left(Q_{n}^{\prime} \alpha-P_{n}^{\prime}\right) & =\alpha Q_{n}\left(\alpha-\frac{P_{n}}{Q_{n}}\right)-Q_{n}^{\prime}\left(\alpha-\frac{P_{n}^{\prime}}{Q_{n}^{\prime}}\right) \\
& =\left(\alpha Q_{n}-Q_{n}^{\prime}\right)\left(\alpha-\frac{P_{n}}{Q_{n}}\right)+Q_{n}^{\prime}\left(\frac{P_{n}^{\prime}}{Q_{n}^{\prime}}-\frac{P_{n}}{Q_{n}}\right),
\end{aligned}
$$

it follows from (5.1), (5.2) and (5.3) that

$$
\begin{aligned}
\left|\alpha^{2} Q_{n}-\alpha Q_{n}^{\prime}-\alpha P_{n}+P_{n}^{\prime}\right| & \ll Q_{n} q_{s_{n}}^{-2} q_{t_{n}}^{-2}+Q_{n}^{-1} \\
& \ll Q_{n}^{-1} .
\end{aligned}
$$

Together with the four linearly independent linear forms with algebraic coefficients

$$
\begin{aligned}
& L_{1}\left(X_{1}, X_{2}, X_{3}, X_{4}\right)=\alpha X_{1}-X_{3}, \\
& L_{2}\left(X_{1}, X_{2}, X_{3}, X_{4}\right)=\alpha X_{2}-X_{4}, \\
& L_{3}\left(X_{1}, X_{2}, X_{3}, X_{4}\right)=\alpha X_{1}-X_{2}, \\
& L_{4}\left(X_{1}, X_{2}, X_{3}, X_{4}\right)=X_{2},
\end{aligned}
$$


introduced in [5], we consider the linear form

$$
L_{5}\left(X_{1}, X_{2}, X_{3}, X_{4}\right)=\alpha^{2} X_{1}-\alpha X_{2}-\alpha X_{3}+X_{4},
$$

and we deduce from (5.1), (5.2), (5.3) and (5.4) that

$$
\prod_{2 \leq j \leq 5}\left|L_{j}\left(Q_{n}, Q_{n}^{\prime}, P_{n}, P_{n}^{\prime}\right)\right| \ll Q_{n}^{2} q_{t_{n}}^{-2} q_{s_{n}}^{-2} \ll q_{r_{n}}^{2} q_{s_{n}}^{-2} .
$$

By (2.3) and (5.3), we have

$$
q_{r_{n}}^{2} q_{s_{n}}^{-2} \ll 2^{-\left|U_{n}\right|} \ll Q_{n}^{-\delta\left(u_{n}+v_{n}-r_{n}\right) /\left(r_{n}+t_{n}\right)},
$$

if $n$ is sufficiently large, where we have set

$$
M=1+\limsup _{\ell \rightarrow+\infty} q_{\ell}^{1 / \ell} \quad \text { and } \quad \delta=\frac{\log 2}{\log M} .
$$

Since a satisfies Condition (\$), we have

$$
\limsup _{n \rightarrow+\infty} \frac{r_{n}}{s_{n}}<1 \text { and } \limsup _{n \rightarrow+\infty} \frac{r_{n}+t_{n}}{s_{n}}<+\infty
$$

thus,

$$
\liminf _{n \rightarrow+\infty} \frac{u_{n}+v_{n}-r_{n}}{r_{n}+t_{n}}>0 .
$$

Consequently, there exists $\varepsilon>0$ such that

$$
\prod_{2 \leq j \leq 5}\left|L_{j}\left(Q_{n}, Q_{n}^{\prime}, P_{n}, P_{n}^{\prime}\right)\right| \ll Q_{n}^{-\varepsilon},
$$

for every sufficiently large $n$.

Following the proof from [5], we apply a first time Theorem 2.1. It implies that the points $\left(Q_{n}, Q_{n}^{\prime}, P_{n}, P_{n}^{\prime}\right)$ lie in a finite union of proper linear subspaces of $\mathbf{Q}^{4}$. As in [5], we deduce that there exists an infinite set of distinct positive integers $\mathcal{N}$ such that $Q_{n}^{\prime}=P_{n}$ for $n$ in $\mathcal{N}$. Thus, for $n$ in $\mathcal{N}$, we have

$$
\left|\alpha^{2} Q_{n}-2 \alpha Q_{n}^{\prime}+P_{n}^{\prime}\right| \ll Q_{n}^{-1},
$$

instead of (5.4). Consider now the three linearly independent linear forms

$$
\begin{aligned}
& L_{1}^{\prime}\left(X_{1}, X_{2}, X_{3}\right)=\alpha^{2} X_{1}-2 \alpha X_{2}+X_{3}, \\
& L_{2}^{\prime}\left(X_{1}, X_{2}, X_{3}\right)=\alpha X_{2}-X_{3}, \\
& L_{3}^{\prime}\left(X_{1}, X_{2}, X_{3}\right)=X_{1} .
\end{aligned}
$$

Evaluating them on the triple $\left(Q_{n}, Q_{n}^{\prime}, P_{n}^{\prime}\right)$ for $n$ in $\mathcal{N}$, it follows from (5.1), (5.3) and (5.5) that

$$
\prod_{1 \leq j \leq 3}\left|L_{j}^{\prime}\left(Q_{n}, Q_{n}^{\prime}, P_{n}^{\prime}\right)\right| \ll Q_{n} q_{t_{n}}^{-2} \ll q_{r_{n}} q_{t_{n}}^{-1} \ll q_{r_{n}} q_{s_{n}}^{-1} \ll Q_{n}^{-\varepsilon / 2},
$$

with the same $\varepsilon$ as above, if $n$ is sufficiently large.

We then apply again Theorem 2.1 and we continue as in the proof of Theorem 2.4 from [5]. We omit the details. 


\section{Concluding remarks}

It is likely that we are now able to get the analogues for continued fraction expansions to all the transcendence results established recently for expansions to an integer base and whose proofs ultimately rest on the Schmidt Subspace Theorem. For instance, combining the arguments of [11] with Theorem 1.3, it is easy to prove that if $1 \leq m<M$ are integers and $\mathbf{a}=a_{1} a_{2} \ldots$ is a word over $\{m, M\}$ such that $\left[0 ; a_{1}, a_{2}, \ldots, a_{\ell}, \ldots\right]$ is algebraic, then there are arbitrarily large (finite) blocks $U$ such that $U^{7 / 3}$ occurs in a.

Recent developments have shown that the use of quantitative versions of the Schmidt Subspace Theorem allows us often to strengthen or to complement results established by means of the qualitative Schmidt Subspace Theorem; see for instance the survey [16]. In particular, by combining ideas from $[6,7,8]$ with new arguments, we have obtained in [17] transcendence measures for transcendental real numbers whose sequence of partial quotients $\mathbf{a}$ is such that $n \mapsto p(n, \mathbf{a}) / n$ is bounded.

Furthermore, proceeding as in [15] and in [18], it seems to be possible to prove that if $\mathbf{a}=a_{1} a_{2} \ldots$ is an infinite word over $\mathbf{Z}_{\geq 1}$ such that $\left[0 ; a_{1}, a_{2}, \ldots, a_{\ell}, \ldots\right]$ is algebraic of degree at least three, then there exists $\delta>0$ such that

$$
\limsup _{n \rightarrow+\infty} \frac{p(n, \mathbf{a})}{n(\log n)^{\delta}}=+\infty
$$

and there exists an effectively computable positive constant $M$ such that

$$
p(n, \mathbf{a}) \geq\left(1+\frac{1}{M}\right) n, \quad \text { for } n \geq 1 .
$$

More details will be given in a subsequent note. Observe that a statement like (6.1) does not contain Theorem 1.1 since there exist infinite words $\mathbf{w}$ such that

$$
\liminf _{n \rightarrow \infty} \frac{p(n, \mathbf{w})}{n}=2 \quad \text { and } \quad \limsup _{n \rightarrow \infty} \frac{p(n, \mathbf{w})}{n^{t}}=+\infty, \quad \text { for any } t>1
$$

see [21].

\section{Acknowledgements}

I am very thankful to the referees for their careful reading and numerous comments, which helped me to improve the presentation of the paper.

$4^{\text {e }}$ SÉRIE - TOME $46-2013$ - No 6 


\section{REFERENCES}

[1] B. Adamczewski, Y. Bugeaud, On the complexity of algebraic numbers. II. Continued fractions, Acta Math. 195 (2005), 1-20.

[2] B. Adamczewski, Y. Bugeaud, Real and $p$-adic expansions involving symmetric patterns, Int. Math. Res. Not. 2006 (2006), Art. ID 75968, 17.

[3] B. Adamczewski, Y. Bugeaud, On the complexity of algebraic numbers. I. Expansions in integer bases, Ann. of Math. 165 (2007), 547-565.

[4] B. Adamczewsi, Y. Bugeaud, On the Maillet-Baker continued fractions, J. reine angew. Math. 606 (2007), 105-121.

[5] B. Adamczewski, Y. Bugeaud, Palindromic continued fractions, Ann. Inst. Fourier (Grenoble) 57 (2007), 1557-1574.

[6] B. Adamczewski, Y. Bugeaud, Mesures de transcendance et aspects quantitatifs de la méthode de Thue-Siegel-Roth-Schmidt, Proc. Lond. Math. Soc. 101 (2010), 1-26.

[7] B. Adamczewski, Y. Bugeaud, Transcendence measures for continued fractions involving repetitive or symmetric patterns, J. Eur. Math. Soc. (JEMS) 12 (2010), 883-914.

[8] B. Adamczewski, Y. Bugeaud, Nombres réels de complexité sous-linéaire : mesures d'irrationalité et de transcendance, J. reine angew. Math. 658 (2011), 65-98.

[9] B. Adamczewsi, Y. Bugeaud, L. Davison, Continued fractions and transcendental numbers, Ann. Inst. Fourier (Grenoble) 56 (2006), 2093-2113.

[10] B. Adamczewski, Y. Bugeaud, F. Luca, Sur la complexité des nombres algébriques, C. R. Math. Acad. Sci. Paris 339 (2004), 11-14.

[11] B. AdAmCZEWSKi, N. RAMPERSAD, On patterns occurring in binary algebraic numbers, Proc. Amer. Math. Soc. 136 (2008), 3105-3109.

[12] J.-P. Allouche, J. L. Davison, M. Queffélec, L. Q. Zamboni, Transcendence of Sturmian or morphic continued fractions, J. Number Theory 91 (2001), 39-66.

[13] J.-P. Allouche, J. Shallit, Automatic sequences. Theory, applications, generalizations, Cambridge Univ. Press, 2003.

[14] A. Baker, Continued fractions of transcendental numbers, Mathematika 9 (1962), 1-8.

[15] Y. Bugeaud, An explicit lower bound for the block complexity of an algebraic number, Atti Accad. Naz. Lincei Cl. Sci. Fis. Mat. Natur. Rend. Lincei (9) Mat. Appl. 19 (2008), 229-235.

[16] Y. Bugeaud, Quantitative versions of the subspace theorem and applications, J. Théor. Nombres Bordeaux 23 (2011), 35-57.

[17] Y. Bugeaud, Continued fractions with low complexity: transcendence measures and quadratic approximation, Compos. Math. 148 (2012), 718-750.

[18] Y. Bugeaud, J.-H. Evertse, On two notions of complexity of algebraic numbers, Acta Arith. 133 (2008), 221-250.

[19] A. Совнам, On the Hartmanis-Stearns problem for a class of tag machines, in Ninth Annual Symposium on Switching and Automata Theory, Schenectady, New York, 1968, 51-60.

[20] A. Совнам, Uniform tag sequences, Math. Systems Theory 6 (1972), 164-192. 
[21] S. Ferenczi, Rank and symbolic complexity, Ergodic Theory Dynam. Systems 16 (1996), 663-682.

[22] G. H. Hardy, E. M. Wright, An introduction to the theory of numbers, fifth ed., The Clarendon Press Oxford Univ. Press, 1979.

[23] E. Maillet, Introduction à la théorie des nombres transcendants et des propriétés arithmétiques des fonctions, Gauthier-Villars, 1906.

[24] M. Morse, G. A. Hedlund, Symbolic Dynamics, Amer. J. Math. 60 (1938), 815-866.

[25] M. Morse, G. A. Hedlund, Symbolic dynamics II. Sturmian trajectories, Amer. J. Math. 62 (1940), 1-42.

[26] O. Perron, Die Lehre von den Kettenbrüchen, Teubner, 1929.

[27] M. QuefFÉLeC, Transcendance des fractions continues de Thue-Morse, J. Number Theory 73 (1998), 201-211.

[28] W. M. Schmidt, Norm form equations, Ann. of Math. 96 (1972), 526-551.

[29] W. M. Schmidt, Diophantine approximation, Lecture Notes in Math. 785, Springer, 1980.

\author{
Yann Bugeaud \\ Université de Strasbourg \\ I.R.M.A. \\ 7, rue René Descartes \\ 67084 Strasbourg Cedex, France \\ E-mail: bugeaud@math.unistra.fr
}

